\title{
A Method of Acquiring Dynamic Road Roughness Based on Vehicle-Road Vertical Coupling
}

\author{
Liu Yongchen", Sun Li, Liang Kun and Xu Lichao
}

Faculty of Transportation Engineering, HuaiYin Institute of Technology, Huai'an 223003, China

\begin{abstract}
A method of acquiring dynamic road roughness was proposed. At first, road elevation data was measured and road surface spectrum was analyzed. Secondly, the simplified interaction dynamic model of quarter vehicle and the EulerBernoulli beam road, visco-elastic Kelvin foundation were built to obtain the wheel dynamic load. In its effect, the beam bending vibration differential equations was built, and road surface vertical displacement solution was acquired by integral transform method under the action of vehicle-road vertical coupling. These were the basis of article study and the important issues. Finally, the dynamic road roughness was analyzed by applying stochastic process theory and the linear superposition method. The conclusion was that the dynamic road roughness was linear superposition on the initial road roughness and road vertical displacement. What was proposed the concept on dynamic road roughness is an important innovation in the vehicle-road dynamics field. This method provided a more accurate road excitation in the course of the vehicle-road dynamic simulation analysis.
\end{abstract}

Keywords: Vehicle-road vertical coupling, dynamic road roughness, quarter vehicle model, road dynamic model, integral transform method.

\section{INTRODUCTION}

In the process of vehicle running, the change of road excitation will be inevitably caused due to the interaction between vehicle and the road. The change of road surface excitation is called dynamic road roughness in this paper, it can accurately predict the interaction between road surface and vehicle dynamic load characteristics. On the one hand, it is an important input of vehicle ride comfort and durability, on the other hand it is an important input of road characteristics' research, this can analyze fatigue characteristics on the ground accurately, so researching vehicle-road coupling of road pavement excitation has important theoretical and engineering significance. In the way of vehicle-road interaction research, many scholars have made important conclusions and method, the literature [1] analyzed the influence of the interaction on car road pavement dynamic characteristics and obtained analytical solution and numerical solution of pavement response. The literature [2-5] studied the cars-pavement sub-grade system dynamics problems, analyzed the impact of different parameters on the dynamic response of pavement, but it didn't offer the influence of the vehicle-road coupling to road roughness. Literature [6-8] simulated road roughness accurately, but it did not consider the pavement deformation caused by road roughness change and did not study the method for determining the dynamic road roughness. Inspired by the previous literature, this paper puts forward vertical-road coupling dynamic access method of road roughness, the method of the dynamic road roughness can provide more accurate pavement incentive for vehicle dynamics simulation and vehicle fatigue durability researches.

\section{ROAD SPECTRUM ACQUISITION TEST}

For typical road roughness, this paper uses road spectrum integrated test system as shown in Fig. (1) and adopts the arrangement check-out car front beam of the laser sensor to eliminate the influence of body posture to the result of the measurement, the acceleration sensor and car arrangement of inertial system are layout on the beam. when testing, totally 9 laser sensors are decorated on the beam, the resolution of which is $0.01 \mathrm{~mm}$, the range of which is $200 \mathrm{~mm}$, the accuracy or bias is $0.1 \%$, the sampling frequency of the wheel track is up to $16 \mathrm{kHz}$. The range of the speed measuring of the system is $20 \mathrm{~km} / \mathrm{h}$ to $120 \mathrm{~km} / \mathrm{h}$. By using the testing system, we take the road roughness measurement on five kinds of typical roads listed in Table 1, Fig. (2) shows a result of a three-level road test.

The roughness measurements of the road are usually the high profile data sequence q on a road, in general, the road roughness which accords with normal distribution and also with ergodicity is stationary random process of each state traversal. Assume that the road without a steep slope, the abnormal damage and so on, we can take the road profile curve as a Gaussian process, statistical characteristics of road surface can be expressed by the power spectrum of surface elevation, the road power spectral density can be expressed as following, 


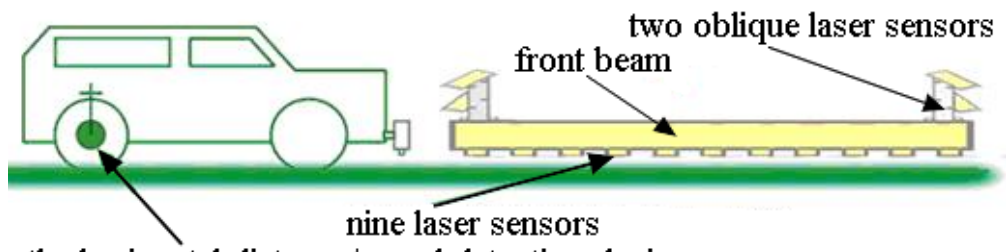

the horizontal distance/speed detection device

Fig. (1). Road roughness test system schematic.

Table 1. Experimental sample sections information.

\begin{tabular}{|c|c|c|}
\hline The Highway Grade & Sample Section & The Length of the Road/m \\
\hline \hline Level one & $\begin{array}{c}\text { LiaoningG304: } \\
\text { Xinmin-A silding door }\end{array}$ & 2809 \\
\hline Level two & $\begin{array}{c}\text { LiaoningS212: } \\
\text { The pine tree-Gaizhou }\end{array}$ & 23546 \\
\hline Level three & $\begin{array}{c}\text { Heilongiang: } \\
\text { Daotaizi-Tuanshanzi }\end{array}$ & 2330 \\
\hline Level four & Jiling: Elm-Birch survey & 250 \\
\hline
\end{tabular}

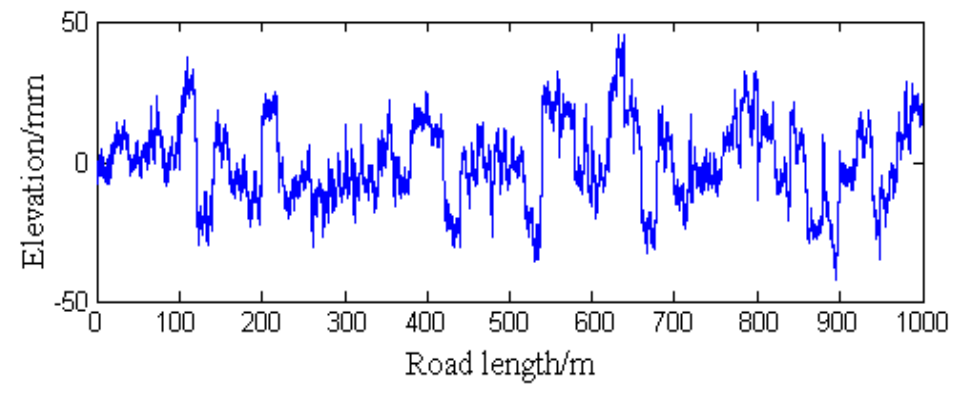

Fig. (2). Measured elevation data of level 3 road.

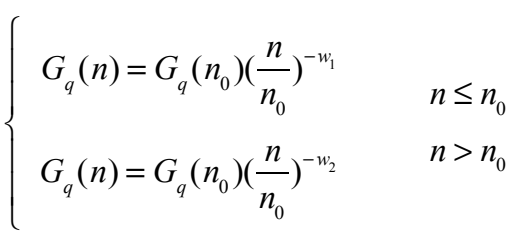

In above type, $n$ is spatial frequency $(1 / \mathrm{m}), \mathrm{n}_{0}=1$ is the reference space frequency $(1 / \mathrm{m}), w_{1}, w_{2}$ is high and low frequency band frequency index, respectively, is the double logarithmic coordinates on the diagonal lines of the slope, the decision of road surface spectrum frequency structure; $G_{q}\left(n_{0}\right)$ is a reference under the spatial frequency of the road power spectral density values $\left(\mathrm{m}^{2} / 1 / \mathrm{m}=\mathrm{m}^{3}\right)$, known as the road roughness coefficient.

When the car drives on the road whose spatial frequency is $n$ on a certain speed of $v n$, that means the road has time frequency, so with time frequency the road surface spectrum is expressed as the type 2 .

$G_{q}(f)=\frac{1}{v} G_{q}(n)$

\section{VEHICLE-ROAD COUPLING DYNAMIC MODEL BUILDING}

\subsection{Coupling Dynamics of a Quarter Car Model}

Most road cars biaxial cars, the road for the four input to it and the actual pavement has isotropic characteristics. We can think that the wheel track around are the same. Without considering the pitch movement the vehicle model can be simplified into a quarter model as shown in Fig. (3). A quarter car model based on the analysis of automotive vertical vibration is reasonable, usually as a research to encourage car response under road roughness model of a vehicle [4].

In the graph, $z_{s}$ is load vertical displacement, $z_{t}$ is the vertical displacement of the sprung, $z_{0}$ is road roughness incentives. $M_{\mathrm{s}}$ is sprung mass, $M_{t}$ is the sprung mass, $K_{s}$ is the suspension stiffness. $K_{t}$ is tire stiffness, $C_{s}$ is suspension damping, $C_{t}$ is tire damping.

By Newton's laws of motion, car model as shown in Fig. (4), can derive the following motion equatio.

$M_{t} \ddot{z}_{t}=K_{t}\left(z_{0}-z_{t}\right)-K_{s}\left(z_{t}-z_{s}\right)-C_{s}\left(\dot{z}_{t}-\dot{z}_{s}\right)-C_{t}\left(\dot{z}_{t}-\dot{z}_{0}\right)$ 


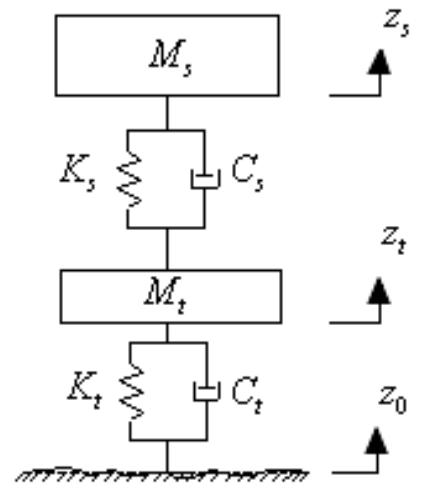

Fig. (3). The quarter vehicle model.

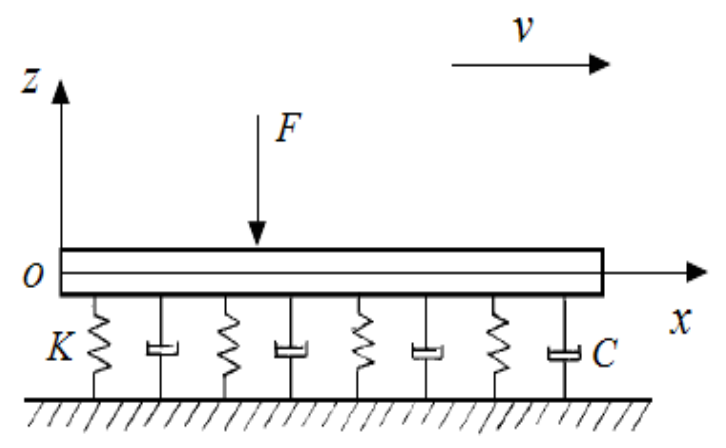

Fig. (4). Simple road model of vehicle-road coupling.

$M_{s} \ddot{z}_{s}=-K_{s}\left(z_{s}-z_{t}\right)-C_{s}\left(\dot{z}_{s}-\dot{z}_{t}\right)$

Road roughness is ergodic Gaussian process. For the smooth process of input, the output of the linear time invariant system is also a smooth process. So motion equation describing the vibration of the system output is a smooth process.

In order to obtain the system frequency response function, if make $z_{0}=Z_{0} e^{i \omega t}$, so establish the system frequency response function matrix.

$\left[\begin{array}{cc}-M_{s} \omega^{2}+K_{s}+i \omega C_{s} & -i \omega C_{s}-K s \\ -i \omega C_{s}-K_{s} & -M_{t} \omega^{2}+K_{t}+K_{s}+i \omega\left(C_{s}+C_{t}\right)\end{array}\right]$
$\left[\begin{array}{c}H_{s}(\omega) \\ H_{t}(\omega)\end{array}\right]=\left[\begin{array}{c}0 \\ K_{t}+i \omega C_{t}\end{array}\right]$

$$
\text { Make } \quad D_{1}=-M_{s} \omega^{2}+K_{s}+i \omega C_{s}, \quad D_{2}=i \omega C_{s}+K_{s},
$$

$D_{3}=-M_{t} \omega^{2}+K_{t}+K_{s}+i \omega\left(C_{s}+C_{t}\right), \quad D_{4}=K_{t}+i \omega C_{t}$, by the laws of Cramer, double frequency response function of the quality system can be obtained.

$$
\begin{aligned}
& H_{s}(\omega)=\frac{D_{2} D_{4}}{D_{1} D_{3}-D_{2}^{2}} \\
& H_{t}(\omega)=\frac{D_{1} D_{4}}{D_{1} D_{3}-D_{2}^{2}}
\end{aligned}
$$

The response power spectral density and motivating power spectral density (PSD) have the following relationships,

$$
\begin{aligned}
& S_{z_{s}}(\omega)=\left|H_{s}(\omega)\right|^{2} S_{z_{0}}(\omega) \\
& S_{z_{t}}(\omega)=\left|H_{t}(\omega)\right|^{2} S_{z_{0}}(\omega)
\end{aligned}
$$

Speed response power spectral density can be obtained through differential as follows,

$$
\begin{aligned}
& S_{\dot{z}_{s}}(\omega)=\omega^{2} S_{z_{s}}(\omega) \\
& S_{\dot{z}_{t}}(\omega)=\omega^{2} S_{z_{t}}(\omega)
\end{aligned}
$$

\subsection{Random Dynamic Load and Statistical Features}

Only consider the vehicle movement in the process of vehicle-road interaction between vertical load and road roughness as the traversal of ergodic random process, resulting in a linear system caused by the vehicle-road load interaction for stationary random load, vehicle-road interaction of dynamic load $\mathrm{F}$ can be represented as type 8 .

$$
F(t)=K_{t} z_{t}(t)+C_{t} \dot{z}_{t}(t)
$$

By type $(6 \mathrm{~b})$, the power spectral density of random dynamic load is shown as type 9 .

$$
S_{F}(\omega)=\left(K_{t}^{2}+C_{t}^{2} \omega^{2}\right)\left|H_{t}(\omega)\right|^{2} S_{z_{0}}(\omega)
$$

\subsection{The Road Model}

The load of vehicles on the road, assuming for flat surface, of point source is taken as the effect of random load, the road is simplified two parts of Kirchhoff plate road pavement and viscoelastic Kelvin foundation [9, 10]. The section of road longitudinal direction, namely, vehicle speed direction, for thickness $h$ of thin plate and elastic and damping elements is shown in Fig. (4). Assuming thickness under moving load is constant and vehicles drive along the road line, namely along the $x$ direction, $x$ is variables and related to the speed of the car. So the quality of the road surface model can be further simplified to the uniform Euler Bernoulli beam. Assumptions includes that the vehicles on the road keep straight at a constant speed, and the tires and the road maintain contacts without jumping out, its quality as the uniform moving vehicles, and beam boundary is simply supported at both ends.

Ignoring the beam damping force and the beam bending vibration differential equation by Dalembert's principle can be established.

$E I \cdot \frac{\partial^{4} z_{r}}{\partial_{x}^{4}}+K z_{r}+C \frac{\partial z_{r}}{\partial t}+m \frac{\partial^{2} z_{r}}{\partial t^{2}}-F(t)=0$

In the type, $z_{r}$ is the vertical displacement, $E$ is pavement modulus of elasticity, $I$ is the section modulus, $m$ is per unit length of the road surface quality, $K$ is reaction modulus of foundation, $C$ is the foundation damping coefficient, $x$ is forward displacement vehicles, $t$ is travel time. 
According to the foregoing assumptions, initial boundary conditions of Euler Bernoulli beam bending equation is as follows.

When $\quad t=0, \quad z_{r}(x, t)=0, \quad \frac{\partial z_{r}}{\partial t}=0, \quad \lim _{t \rightarrow \pm \infty} \frac{\partial^{(n)} z_{r}}{\partial t^{n}}=0$, ( $n=0,1,2,3)$, transforming type variable $t$ for Laplace, and type 10 is changed the type 11 .

$E I \frac{d^{4} \tilde{z}_{r}}{d x^{4}}+K \tilde{z}_{r}+C s \tilde{z}_{r}+m s^{2} \tilde{z}_{r}=\tilde{F}(s)$

Transforming type variable $x$ for Fourier,

$E I \omega^{4} \overline{\tilde{z}}_{r}+K \overline{\tilde{z}}_{r}+C s \overline{\tilde{z}}_{r}+m s^{2} \overline{\tilde{z}}_{r}=\overline{\tilde{F}}(s)$

That is,

$\overline{\tilde{z}}_{r}=\overline{\tilde{F}}(s)\left(m s^{2}+C s+E I \omega^{4}+K\right)^{-1}$

Using the Laplace inverse transformation, at the same time, using the convolution theorem, we can get the following.

$\bar{z}_{r}=\bar{F}(t) \times L^{-1}\left[\left(s^{2}+\frac{C}{m} s+\frac{E I}{m} \omega^{4}+\frac{K}{m}\right)^{-1}\right]$

Making,

$\left\{\begin{array}{l}a=\frac{C}{m} \\ b=\frac{1}{4 m} \sqrt{C^{2}+4 m E I \omega^{4}+4 m K}\end{array}\right.$

By Laplace transform table, the type 16 is available.

$\bar{z}_{r}=\bar{F}(t) \times\left[\frac{1}{2 b}\left\{\exp \left[\left(b-\frac{a}{2}\right) t\right]-\exp \left[\left(-b-\frac{a}{2}\right) t\right]\right\}\right]$

Defined by the convolution is obtained.

$\bar{z}_{r}=\int_{-\infty}^{+\infty} \bar{F}(\tau)\left\{\frac{1}{2 b}\left[\exp \left(b-\frac{a}{2}\right)(t-\tau)-\exp \left(-b-\frac{a}{2}\right)(t-\tau)\right]\right\} d \tau$

To Fourier inverse transformation on above type, we can obtain the parameter of $z_{r}$.

$$
\begin{aligned}
z_{r} & =\frac{1}{4 \pi} \int_{-\infty}^{+\infty} \int_{-\infty}^{+\infty} \frac{1}{b} \bar{F}(\tau)\left\{\exp \left[\left(b-\frac{a}{2}\right)(t-\tau)+i \omega x\right]\right. \\
& \left.-\exp \left[\left(-b-\frac{a}{2}\right)(t-\tau)+i \omega x\right]\right\} d \tau d \omega
\end{aligned}
$$

By the analytic the formula of road surface vertical displacements on the conditiong to interaction load between the moving vehicle and the road pavement, road pavement vertical displacement is obtained with a numerical calculation method of random data.

\section{DYNAMIC ROAD ROUGHNESS}

The original surface roughness $z_{0}$ is related to a benchmark of road surface elevation, it is objective existence and

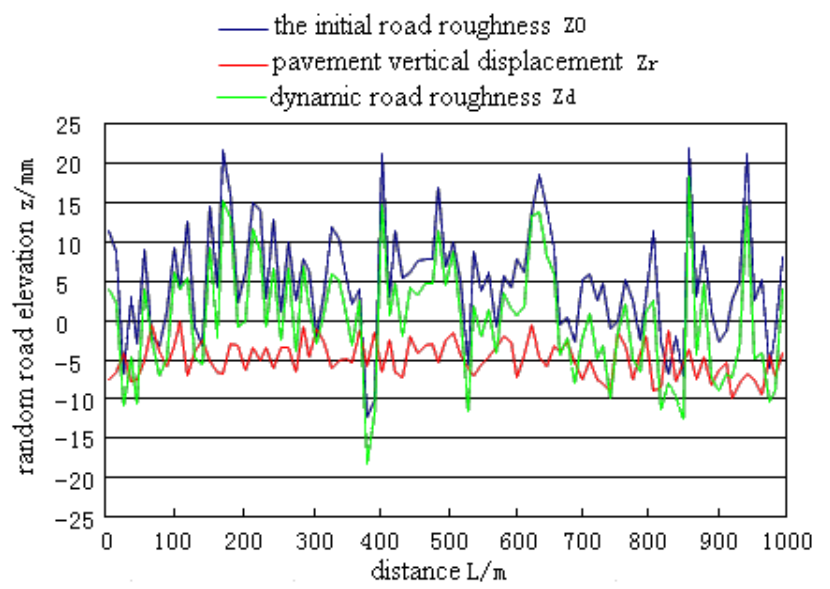

Fig. (5). Dynamic road roughness.

stationary Gaussian process. And road surface vertical displacement value $z_{r}$ is under the wheel with motor load, as a random process. For judging the stability, making $\beta=t-\tau$, the vertical response $z_{r}$ of pavement structure beam of the mean $E\left[z_{r}(t)\right]$ and autocorrelation function $R_{z}\left(t_{1}, t_{2}\right)$ can be represented.

$$
\begin{aligned}
E\left[z_{r}(t)\right]= & \frac{1}{4 \pi} F(t) \int_{-\infty}^{+\infty} \int_{-\infty}^{+\infty} \frac{1}{b}\left\{\operatorname { e x p } \left[\left(-b-\frac{a}{2}\right) \beta\right.\right. \\
& \left.+i \omega v t]+\exp \left[\left(b-\frac{a}{2}\right) \beta+i \omega v t\right]\right\} d \beta d \omega \\
R_{z}\left(t_{1}, t_{2}\right)= & E\left[z_{r}\left(t_{1}\right) z_{r}\left(t_{2}\right)\right] \\
= & \frac{1}{16 \pi^{2}} \int_{-\infty}^{+\infty} \int_{-\infty}^{+\infty} \frac{1}{b^{2}} R_{F}\left(t_{1}, t_{2}\right) \exp i \omega v\left(t_{1}-t_{2}\right) \\
& \left\{\exp \left[\left(b-\frac{a}{2}\right)\left(\beta_{1}+\beta_{2}\right)\right]+\exp \left[\left(-b-\frac{a}{2}\right)\left(\beta_{1}+\beta_{2}\right)\right]\right. \\
& \left.-\exp \left[\left(b-\frac{a}{2}\right) \beta_{1}-\left(-b-\frac{a}{2}\right) \beta_{2}\right)\right] \\
& \left.\left.+\exp \left[\left(-b-\frac{a}{2}\right) \beta_{1}+\left(b-\frac{a}{2}\right) \beta_{2}\right)\right]\right\} d \beta d \omega
\end{aligned}
$$

In type 19 and 20, the mean value is a function of time $t$, autocorrelation function is associated with $t_{1}, t_{2}$, so the vehicle movement and the surface vertical displacement $z_{r}$ generated by dynamic load is non-stationary process, it is not suitable for direct description with the power spectral density but piecewise smooth processing can be performed.

At a constant speed, vehicle displacement is proportional to the time, that is $x=v t$, it can be determined that the road beam structure maximum vertical displacement position at a point in the vehicle dynamic load point, namely, $z_{r}=z_{r}(t)$. Thus determination of road roughness is dynamic, it can be expressed as a superposition of linear algebra of an initial relative to a datum road roughness $z_{0}$ and road vertical displacement $z_{r}$, it is shown in Fig. (5), dynamic road roughness can be obtained $z_{d}=z_{0}+z_{r}$. 


\section{CONCLUSION}

By road roughness measurement test and road surface spectrum analysis, we put forward dynamic access method of road roughness and basically have the following conclusion.

(1) Using the $1 / 4$ vehicle model and by Euler Bernoulli beam pavement, viscoelastic Kelvin foundation constitute a simplified road model, vehicle road vertical coupling dynamics model is established and analyzed the wheel dynamic load of initial road excitation.

(2) We set up the car road coupling beams under the action of bending vibration differential equations, integral transform method is used for road surface vertical displacement of the analytical solution and analyzed the characteristics of random then proving it is the non-stationary random process.

(3) Using the theory of stochastic process to determine the road excitation substance is the dynamic road roughness under the action of vertical-road coupling, it can be said for the initial vertical displacement of road roughness and pavement of the linear superposition.

\section{CONFLICT OF INTEREST}

The author confirms that this article content has no conflict of interest.

\section{ACKNOWLEDGEMENTS}

The authors gratefully acknowledge the sponsored by the supports from Six Kinds Talent Summit Project of Jiangsu Province (2014-JXQC-007), and Qing Lan Project of Jiangsu
Province, and the Natural Science Foundation of the Jiangsu Higher Education Institutions of China (13KJB580001).

\section{REFERENCES}

[1] D. X. Jun, and S. Lu, Study on dynamics of vehicle-ground pavement structure system, Beijing: China Communications Press, 2000

[2] L. S. Hua, Y. S. Pu, and L. H.Yu, "Dynamical response and parameters analysis for vehicle-pavement-foundation system", Journal of Beijing Jiaotong University, vol. 34, pp. 127-5, April, 2010.

[3] L. D. Wei, L. G. Zheng, and C. H. Ming, "Dynamic response of pavement based on random dynamic load of vehicle", Transactions of the Chinese Society for Agricultural Machinery, vol. 42, pp.28-6, February, 2011.

[4] Z. Y. Min, T. Z. Ming, and L. B. Ying, "Quarter vehicle-road coupling dynamics models", Journal of Tong Ji University (Natural Science), vol. 40, pp. 408-6, March, 2012.

[5] Z. B. Qiang, and L. Liang, "Dynamic model and simulation of a vehicle-road coupled system", Journal of Vibration and Shock, vol. 29, pp. 35-4, February, 2010.

[6] Z. L. Ping, and G. L. Xin, "Identification of random road spectrum based on inverse pseudo-excitation method", Journal of Northeastern University (Natural Science), vol. 33, pp. 258-4, February, 2012.

[7] L. X. Dong, D. Z. Dang, and G. Feng, "Study of simulation of road roughness based on inverse transform", China Journal of Highway and Transport, no. 1, pp. 122-5, January, 2005.

[8] H. Y. Tao, W. G. Lin, and L. Jian, "Design and implementation of the 3D virtual road reconstruction system for vehicle", Chinese Journal of Mechanical Engineering, vol. 87, pp. 126-8, August, 2011.

[9] Y. Yu, T. N. Guan, and H. X. Chun, "Vibration analysis of a simply supported beam traversed by uniform distributed moving mass", Journal of Vibration and Shock, Vol.24, pp. 19-4, March, 2005.

[10] L. D. Wei, L. Wei, and C. H. Ming, "Dynamic load characteristics of heavy vehicle based on multi-body dynamic model", Transactions of the Chinese Society for Agricultural Machinery, vol. 40, pp. 7-6, December, 2009.

Received: November 25, 2014

Revised: January 07, 2015

Accepted: January 20, 2015

(C) Yongchen et al.; Licensee Bentham Open.

This is an open access article licensed under the terms of the Creative Commons Attribution Non-Commercial License (http://creativecommons.org/licenses/by-nc/3.0/) which permits unrestricted, non-commercial use, distribution and reproduction in any medium, provided the work is properly cited. 